\title{
THE CLASS NUMBER OF THE FIELD OF $5^{n}$ TH ROOTS OF UNITY
}

\author{
LAWRENCE C. WASHINGTON ${ }^{1}$
}

\begin{abstract}
Let $h_{n}^{-}$be the relative class number of the field of $5^{n}$ th roots of unity. If $l$ is any prime number, then the $l$-part of $h_{n}^{-}$is bounded independent of $n$.
\end{abstract}

Let $k$ be a number field, $K / k$ the cyclotomic $\mathbf{Z}_{p}$-extension of $k$, and $k_{n}$ the unique intermediate field of degree $p^{n}$ over $k$. In [4] it was conjectured that if $l \neq p$ is any prime number then the $l$-part of the class number of $k_{n}$ is bounded independent of $n$. This conjecture arose from analogy with the case of function fields over finite fields, where $\mathbf{Z}_{p}$-extensions can be obtained by extending the field of constants. In this case it is not difficult to show that the $l$-part of the order of the group of divisor classes of degree zero is bounded independent of $n[4]$.

For number fields, the conjecture has been proved when $k / \mathbf{Q}$ is abelian and $p=2$ or 3 . The main obstacle for larger primes $p$ is the existence of $p$-adic $(p-1)$ st roots of unity, which are, of course, harder to handle when $p \geqslant 5$. In this note we attack the case of the simplest $\mathbf{Z}_{5}$-extension, namely the one obtained by adjoining all $5^{n}$ th roots of unity, for all $n \geqslant 1$, to the field of 5 th roots of unity.

Recall that for any imaginary abelian number field $K$ there is a maximal real subfield $K^{+}$, and since $K / K^{+}$is totally ramified (at $\infty$ ) the class number $h^{+}$of $K^{+}$divides the class number $h$ of $K$. The quotient $h / h^{+}$is called the relative class number $h^{-}$.

THEOREM. Let $h_{n}^{-}$be the relative class number of $\mathbf{Q}\left(\zeta_{5^{n}}\right)$, where $\zeta_{5^{n}}$ is a primitive $5^{n}$ th root of unity. Let $l$ be any prime number and let $l^{e_{n}^{-}} \| h_{n}^{-}$. Then $e_{n}^{-}$ is bounded as $n \rightarrow \infty$. In fact, if $m_{l}$ is determined by $5^{m_{l}} \| l^{4}-1$ and $n \geqslant 2 m_{l}+$ 2 , then $l \nmid h_{n}^{-} / h_{n-1}^{-} .\left(l^{a} \| b\right.$ means $\left.l^{a} \mid b, l^{a+1} \nmid b\right)$.

Proof. Since 5 is a regular prime, $5 \nmid h_{n}^{-}$for any $n$ [2]. Therefore, we assume $l \neq 5$.

The set of odd Dirichlet characters of $\mathbf{Q}\left(\zeta_{5^{n}}\right)$ is obtained as follows: Let $\chi_{1}$, $\chi_{2}$ be the odd characters for $\mathbf{Q}\left(\zeta_{5}\right) / \mathbf{Q}$ and let $\psi_{n}$ be a character of conductor $5^{n}$ such that $\psi_{n}(a)$ depends only on $a^{4} \bmod 5^{n}$ (therefore $\psi_{n}$ generates the

Received by the editors March 1, 1976.

AMS (MOS) subject classifications (1970). Primary 12A50, 12 A35.

${ }^{1}$ Partially supported by NSF Grant MPS74-07491A01. 
characters of the subfield of $\mathbf{Q}\left(\zeta_{5^{n}}\right)$ of degree $5^{n-1}$ over $\left.\mathbf{Q}\right)$. The odd characters of $\mathbf{Q}\left(\zeta_{5^{n}}\right)$ are then $\left\{\chi_{i} \psi_{n}^{j}\right\}$, where $i=1,2$ and $0 \leqslant j \leqslant 5^{n-1}-1$. Since $\chi_{i} \psi_{n}^{j}$ is also a character for $\mathbf{Q}\left(\zeta_{5^{n-1}}\right)$ if $5 \mid j$, we obtain from the analytic class number formula [1]:

$$
\frac{h_{n}^{-}}{h_{n-1}^{-}}=5 \cdot \prod_{i=1}^{2} \prod_{j=1 ; 5 \nmid j}^{5^{n-1}-1}\left(-\frac{1}{2} B_{1, \chi_{i} \psi_{n}}\right),
$$

where

$$
B_{1 . \chi_{\psi_{n}^{j}}}=\frac{1}{5^{n}} \sum_{0<a<5^{n}} a \chi_{i} \psi_{n}^{j}(a) .
$$

Let $F_{m}=\mathbf{Q}\left(\sqrt{-1}, \zeta_{5^{m}}\right)$. Note that $B_{1 . \chi_{\psi}^{\prime} \psi_{n}^{\prime}} \in F_{n}$. For positive integers $n, m$ with $n \geqslant 2 m$, let $\operatorname{Tr}$ be the trace function from $F_{n}$ to $F_{m}$. We shall calculate $\operatorname{Tr}\left(\frac{1}{2} B_{1, \chi \psi_{n}}\right)$, where $\chi=\chi_{1}$ or $\chi_{2}$. Note that $\operatorname{Tr}\left(\zeta_{5^{c}}\right)=0$ if $c>m$. Therefore,

$$
\begin{aligned}
\operatorname{Tr}\left(\psi_{n}(a)\right) & \neq 0 \Leftrightarrow \psi_{n}(a)^{5^{m}}=1 \Leftrightarrow \psi_{n}\left(a^{5^{m}}\right)=1 \\
& \Leftrightarrow a^{4 \cdot 5^{m}} \equiv 1 \bmod 5^{n} \Leftrightarrow a^{4} \equiv 1 \bmod 5^{n-m},
\end{aligned}
$$

in which case $\operatorname{Tr}\left(\psi_{n}(a)\right)=5^{n-m} \psi_{n}(a)$. Therefore,

$$
\operatorname{Tr}\left(\frac{1}{2} B_{1, \chi \psi_{n}}\right)=\frac{1}{2 \cdot 5^{m}} \sum_{0<a<5^{n} ; a^{4} \equiv 1\left(5^{n-m}\right)} a \chi \psi_{n}(a) .
$$

Let $b$ satisfy $b^{4} \equiv 1 \bmod 5^{n-m}, 1<b<\frac{1}{2} 5^{n-m}$ (so $b \equiv 2$ or $3 \bmod 5$ ). Then the sum becomes

$$
\frac{1}{2 \cdot 5^{m}} \sum_{a \equiv \pm 1\left(5^{n-m}\right)} a \chi \psi_{n}(a)+\frac{1}{2 \cdot 5^{m}} \sum_{a \equiv \pm b\left(5^{n-m}\right)} a \chi \psi_{n}(a) .
$$

We now evaluate $\sum_{a \equiv \pm c\left(5^{n-m}\right)} a \chi \psi_{n}(a)$, where $0<c<5^{n-m}$ and $5 \nmid c$. First, note that

$$
\sum_{0<k<5^{m}} \psi_{n}\left(c+k 5^{n-m}\right)=\psi_{n}(c) \sum \psi_{n}\left(1+c^{-1} k 5^{n-m}\right)=0,
$$

since the latter sum is over the subgroup $\left(1+5^{n-m} \mathbf{Z}\right) /\left(1+5^{n} \mathbf{Z}\right)$ of $\left(\mathbf{Z} / 5^{n} \mathbf{Z}\right)^{x}$. Therefore, using the fact that $\chi$ is an odd character of conductor 5 and $\psi_{n}$ is an even character, we obtain

$$
\begin{gathered}
\sum_{t=0}^{5^{m}-1}\left(c+5^{n-m} t\right) \chi \psi_{n}\left(c+5^{n-m_{t}}\right)+\left(5^{n}-c-5^{n-m} t\right) \chi \psi_{n}\left(5^{n}-c-5^{n-m_{t}} t\right) \\
=2 \chi \psi_{n}(c) \cdot 5^{n-m} \sum_{t=0}^{5^{m}-1} t \psi_{n}\left(1+c^{-1} t 5^{n-m}\right) .
\end{gathered}
$$

Let $\zeta=\psi_{n}\left(1+5^{n-m}\right)$, so $\zeta$ is a primitive $5^{m}$ th root of unity. Then, since $n \geqslant 2 m$, we have

$$
\left(1+5^{n-m}\right)^{r} \equiv 1+\imath 5^{n-m} \bmod 5^{n}
$$

so we obtain 


$$
\begin{aligned}
\sum_{a \equiv \pm c\left(5^{n-m}\right)} a \chi \psi_{n}(a) & =2 \cdot \chi \psi_{n}(c) 5^{n-m} \sum_{t=0}^{5^{m}-1} t \zeta^{c^{-1} t} \\
& =2 \chi \psi_{n}(c) \cdot 5^{n} \cdot 1 /\left(\zeta^{c^{-1}}-1\right) .
\end{aligned}
$$

Therefore,

$$
5^{n-m} \operatorname{Tr}\left(\frac{1}{2} B_{1, \chi \psi_{n}}\right)=\frac{1}{\zeta-1}+\frac{\chi \psi_{n}(b)}{\zeta^{b^{-1}}-1}=\frac{1}{\zeta-1}+\frac{\chi(b) \zeta^{a}}{\zeta^{b^{-1}}-1}
$$

for some integer $a$, since $b^{4} \equiv 1 \bmod 5^{n-m}$ implies that $\psi_{n}(b)^{5^{m}}=1$.

Now, suppose $n \geqslant 2 m_{l}+2$ and that $l \mid h_{n}^{-} / h_{n-1}^{-}$. Then there is a prime $\tilde{l}$ over $l$ such that $\tilde{l} \mid \frac{1}{2} B_{1, \chi \psi_{n}^{j}}$ for some $\chi=\chi_{1}$ or $\chi_{2}$ and some $j$ with $5 \nmid j$. By changing the original choice of $\psi_{n}$ if necessary, we may assume that $\tilde{l} \mid \frac{1}{2} B_{1, \chi \psi_{n}}$. The definition of $m_{l}$ implies that all primes lying above $l$, in particular $\tilde{l}$, are inert for $F_{n} / F_{m_{i}}$. If we let $m=m_{l}+1$ (therefore $n \geqslant 2 m$ ), we find that $\tilde{l} \mid 5^{m-n} \operatorname{Tr}\left(\frac{1}{2} B_{1, \chi \psi_{n}}\right)$. Now let $\sigma \in \operatorname{Gal}\left(F_{m} / F_{m_{l}}\right)$ be defined by $\sigma\left(\zeta_{5^{m}}\right)=\zeta_{5^{m}}^{j}$, where $j \equiv 1 \bmod 5^{m_{l}}$. Since $\tilde{l}$ is inert for $F_{m} / F_{m_{l}}$ it follows that

$$
\tilde{l} \mid \sigma\left(5^{m-n} \operatorname{Tr}\left(\frac{1}{2} B_{1, \chi \psi_{n}}\right)\right)=\frac{1}{\zeta^{j}-1}+\frac{\chi(b) \zeta^{a j}}{\zeta^{j b^{-1}}-1} .
$$

Let $\alpha=\zeta^{5^{m_{l}}}$. Then we obtain (let $j=1+u 5^{m_{l}}$ )

$$
\zeta^{b^{-1}} \alpha^{u b^{-1}}-1+\chi(b) \zeta^{a+1} \alpha^{u(a+1)}-\chi(b) \zeta^{a} \alpha^{u a} \equiv 0 \bmod \tilde{l}
$$

for all integers $u$. By linear independence of characters [3, p. 209], or Vanermonde determinants, we see that the coefficients of each power of $\alpha$ must vanish $\bmod \tilde{l}$. The only way this can happen is to have $b^{-1}, 0, a+1$, and $a$ satisfy either $0 \equiv a+1, b^{-1} \equiv a \bmod 5$ or $0 \equiv a, b^{-1} \equiv a+1 \bmod 5$ ( since $\alpha^{5}=1$; note that $b^{-1} \equiv 0$ cannot happen because of the choice of $b$ ). Therefore, either $b^{-1} \equiv-1 \bmod 5$ or $b^{-1} \equiv 1 \bmod 5$. But neither of these can happen since $b \equiv 2$ or $3 \bmod 5$. We have therefore obtained a contradiction; consequently we must have $l+h_{n}^{-} / h_{n-1}^{-}$. Q.E.D.

We remark that using the action of the Galois group plus linear independence of characters is essentially equivalent to multiplying the expression by an arbitrary root of unity and applying the trace function. This latter method was employed in [4, p. 180]. The calculations are the same in both cases. In fact, the trace function could have been used in the last step of the above proof, with the same result.

Finally, we note that the above theorem unfortunately does not seem to imply the corresponding result for the full class number $h_{n}$, since the usual techniques for using $h_{n}^{-}$to obtain results about $h_{n}^{+}$require that the $l$ th roots of unity be in the field being considered (see [4]). 


\section{REFERENCES}

1. H. Hasse, Über die Klassenzahl abelscher Zahlkörper, Akademie-Verlag, Berlin, 1952. MR 14, 141.

2. K. Iwasawa, A note on class numbers of algebraic number fields, Abh. Math. Sem. Univ. Hamburg 20 (1956), 257-258. MR 18, 644.

3. S. Lang, Algebra, Addison-Wesley, Reading, Mass., 1965. MR 33 \#416.

4. L. Washington, Class numbers and $\mathbf{Z}_{p}$-extensions, Math. Ann. 214 (1975), 177-193. MR 51 \#437.

Department of Mathematics, Stanford University, Stanford, California 94305 